\title{
Reduced CD38 expression on CD34+ cells as a diagnostic test in myelodysplastic syndromes
}

\author{
Nicolas Goardon, ${ }^{1}$ Emmanouil Nikolousis, ${ }^{2}$ Alexander Sternberg, ${ }^{1-3}$ Wai-Kit Chu, ${ }^{4}$ Charles Craddock, ${ }^{2}$ Peter Richardson, \\ Richard Benson, ${ }^{5}$ Mark Drayson, ${ }^{7}$ Graham Standen, ${ }^{6}$ Paresh Vyas, ${ }^{1}$ and Sylvie Freeman ${ }^{2,7}$ \\ ${ }^{1}$ MRC Molecular Haematology Unit and Department of Haematology, Weatherall Institute of Molecular Medicine, John Radcliffe \\ Hospital, Oxford; '2Department of Haematology, University of Birmingham and University Hospital of Birmingham, Birmingham; \\ ${ }^{3}$ Department of Haematology Great Western Hospital, Swindon; ${ }^{4}$ Cancer Research UK, Weatherall Institute of Molecular Medicine, \\ John Radcliffe Hospital, Oxford; ${ }^{5}$ Nuffield Department of Orthopaedics, Nuffield Orthopaedic Hospital, Oxford; ${ }^{6}$ Department of \\ Haematology, Bristol Royal Infirmary, Bristol, and ${ }^{7}$ Department of Immunology, University of Birmingham and University Hospital \\ of Birmingham, Birmingham, UK
}

\section{ABSTRACT}

Diagnosis of myelodysplastic syndrome can be difficult especially in cases with a low blast count and a normal karyotype. Flow cytometry has been used to distinguish myelodysplastic syndrome from non-clonal cytopenias. No one single simple flow cytometric parameter has been proposed to be diagnostic of myelodysplastic syndrome. We have studied samples from 100 myelodysplastic syndrome patients and as control samples; 70 non-clonal cytopenias, 5 subjects with normal hematology, 31 patients with acute myeloid leukemia and 11 with chronic myelomonocytic leukemia or myeloproliferative disorder. We show that reduced relative mean fluorescence of $\mathrm{CD} 38$ below a threshold value on $\mathrm{CD} 34^{+}$cells diagnosed low-grade myelodysplastic syndrome with $95 \%$ sensitivity $(95 \%$ confidence interval, $87-99 \%$ ) and $92 \%$ specificity (95\% confidence interval, $82-97 \%$ ). This simple flow cytometric test may be of value in the routine clinical diagnosis of myelodysplastic syndrome, especially in cases with a low blast count and normal karyotype.

Key words: myelodyplasia, immunophenotype, FACS diagnosis.

Citation: Goardon N, Nikolousis E, Sternberg A, Chu W-K, Craddock C, Richardson P, Benson R, Drayson M, Standen G, Vyas $P$ and Freeman S. Reduced CD38 expression on CD $34^{+}$ cells as a diagnostic test in myelodysplastic syndromes. Haematologica 2009;94:1160-1163.

doi:10.3324/haematol.2008.004085

(C)2009 Ferrata Storti Foundation. This is an open-access paper.

\section{Introduction}

The clonal myelodysplastic syndromes (MDS) cause cytopenias that are more prevalent in an ageing population. ${ }^{1,2}$ Low-risk MDS (blast count $<5 \%$ ) without ring sideroblasts and a normal karyotype can be difficult to differentiate morphologically from dysplastic non-clonal cytopenias, that are also common in the elderly. Several studies have used flow cytometry to try and distinguish low-risk MDS from non-clonal cytopenias by assessing the pattern of myeloid/erythroid/lymphoid maturation from progenitors and the immunophenotype of the $\mathrm{CD}_{3} 4^{+}$compartment. ${ }^{3-13}$ However, no one simple flow-based marker reliably differentiates $\mathrm{MDS}$ from non-clonal cytopenias. This has given rise to a number of scoring systems combining multiple measurements. They have variable sensitivity/specificity in MDS diagnosis and can be complex, which detracts from their routine use in clinical non-specialist flow cytometry laboratories.

Perhaps the most common abnormality that we, ${ }^{6}$ and others $9,11,12,14,15$ have documented are reduced numbers of B-cell $\mathrm{CD}_{3} 4^{+}$progenitors in MDS. As B-cell progenitors have higher CD38 expression, and thus a higher CD38 relative mean fluorescent intensity (RFMI) as a population, we hypothesized that a lower number of B-cell progenitors would reduce the mean fluorescence intensity (MFI) of CD38 expression on $\mathrm{CD}_{34}{ }^{+}$cells.

In addition, the MFI of CD38 on $\mathrm{CD} 34^{+}$cells would be further reduced in high-risk $M D S$ as this condition has a higher number of immature $\mathrm{CD} 34^{+} \mathrm{CD} 38^{-}$hemopoietic progenitors. ${ }^{8}$ Thus, we investigated whether reduced mean fluorescence intensity (MFI) of CD38 expression on $\mathrm{CD}^{4} 4^{+}$cells could be used as a surrogate marker for abnormalities in the MDS CD34+ compartment and whether this would provide a simple useful single flow cytometric measurement diagnostic of MDS.

The online version of this article contains a supplementary appendix. Acknowledgments: the authors thank all the hospitals and teams that are part of the UK NCRN MDSBio collaboration. We thank Professor Andrew Carr and the staff and patients of all the Departments contributing to the MDSBio study for facilitating the collection of normal bone marrow samples and samples from patients. We thank Esther Rose-Edlundh for processing bone marrow samples. Funding: NG was supported by a European Molecular Biology organisation long-term fellowship and MRC funding. PV acknowledges funding by the MRC. This work was also funded by the Oxford Partnership Comprehensive Biomedical Research Centre with funding from the Department of Health's NIHR Biomedical Research Centres funding scheme.

Manuscript received on November 26, 2008. Revised version arrived on March 11, 2009. Manuscript accepted on March 18, 2009. Correspondence: Sylvie Freeman, Departments of Haematology and Immunology, University of Birmingham and University Hospital of Birmingham UK and Paresh Vyas, MRC Molecular Haematology Unit and Department of Haematology, Weatherall Institute of Molecular Medicine, John Radcliffe Hospital, Oxford OX3 9DU, UK. E-mail: s.freeman@bham.ac.uk 


\section{Design and Methods}

\section{Patients}

Human adult bone marrow was obtained after informed consent. Two separate sample cohorts were analyzed in 2 different laboratories. Patients' characteristics are shown in Online Supplementary Tables S1 and S2.

\section{Flow cytometry}

All bone marrow samples were processed within 24$48 \mathrm{~h}$ of aspiration. For cohort 1 samples, mononuclear cells were isolated by layering cells on Ficoll (Lymphoprep, Axisshield UK), followed by centrifugation at $1800 \mathrm{rpm}$ for 30 min. Mononuclear cells were labeled with; FITC-conjugated anti-CD45 (clone HI30), PECy5-conjugated anti-IgG1, APC-conjugated anti-CD34 (581) (Becton Dickinson, San Josè, CA, USA); PECy5 anti-CD19 (J3-119), PECy5 antiCD38 (LS198-4-3) (Beckman Coulter, Fullerton, CA, USA). In cohort 2 , nucleated cells were analyzed after red cell lysis. They were labeled with combinations of; PERCPconjugated anti-CD45 (clone 2D1); PE-conjugated antiCD38 (HB7), APC-conjugated anti-CD34 (8G12), PERCPconjugated anti-CD34 (8G12), PE-conjugated isotype control (CD56-MY31), APC-conjugated anti-CD33 (P67.6), PE-conjugated anti-CD117 (10452) (Becton Dickinson, San Jose, CA). In both cohorts cells were analyzed on a FACSCalibur flow cytometer (Becton Dickinson, San José, CA, USA). At least 100,000 nucleated cells (cohort 1) or 20,000 nucleated cells (cohort 2) were acquired for each antibody combination. All procedures were completed within 24-48 h of marrow aspiration for every patient in both cohort 1 and 2 . This time period is less than the recommended upper limit of cell sample storage for FCM (72 h). Data was further analyzed using FlowJo software (Treestar, Ashland, OR, USA). CD34+ cells were defined by standard serial gating (CD34 ${ }^{+} \mathrm{SC}^{\text {lo }}$ followed by $\left.\mathrm{CD} 34^{+} \mathrm{CD} 45^{\circ}\right)$ (Online Supplementary Figure S1). Samples from cohort 2 with insufficient $\mathrm{CD} 34^{+}$cells were excluded, as measurements from analysis would be unreliable. It is important that sufficient numbers of nucleated cells/CD34+ cells are collected in hypocellular samples so that samples from hypoplastic MDS and aplastic anemia patients can be studied. RMFI was quantified as CD38 mean fluorescence intensity (MFI) divided by MFI of isotype control staining (Online Supplementary Figure S1). Samples from the second cohort were processed in a single clinical laboratory by at least 3 operators in rotation all using standardized operating procedures for instrument set up and compensation. Gating analysis was then checked by a further operator $(S F)$. The intra-assay coefficient of variation $(\mathrm{CV})$ of $\mathrm{CD} 38$ mean fluorescence intensity on $\mathrm{CD}_{4} 4^{+}$cells in the clinical laboratory processing samples from the second cohort was $8.5 \%$ (10 replicates of a single sample) when collecting 20,000 nucleated bone marrow cells ( $200 \mathrm{CD} 34^{+}$cells on average). This would be further improved by collecting sufficient nucleated cells to acquire more $\mathrm{CD} 34^{+}$cells. Gating $\mathrm{CD} 34^{+}$cells is routinely performed in many laboratories but in view of potential interoperator and inter-laboratory variability, as well as variation of other flow cytometry parameters (instrument, fluorochrome, sample anticoagulation/ manipulation), other laboratories should establish their own reference ranges using the appropriate standardized analysis protocol when performing this assay.

\section{Results and Discussion}

We initially compared $\mathrm{CD} 34^{+}$cells from low-grade MDS patients $(n=10)$, normal controls $(n=5)$, pathological controls and regenerating bone marrow samples post-allogeneic bone transplant $(\mathrm{n}=10)$ (Online Supplementary Figure S2 and Online Supplementary Table S1). In this cohort, essentially a training cohort, we specifically studied MDS cases where the diagnosis was unambiguous and controls that could not be confused with MDS. CD38 MFI was quantified by RMFI (see Methods) as this measurement is more

$A$

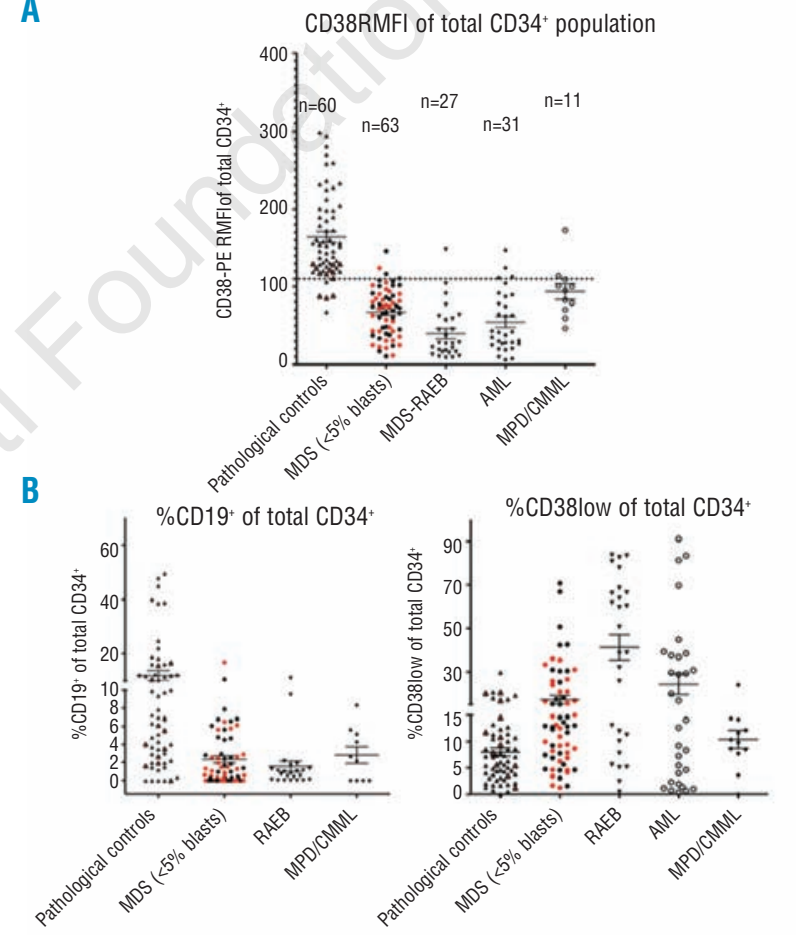

Figure 1. CD38 Relative Mean Fluorescence Intensity (RMFI) of $\mathrm{CD}^{+} 4^{+}$cells and percentages of $\mathrm{B}$ cell and CD38 ${ }^{\text {low }}$ progenitors in MDS patients and control populations. Graphs show: (A) CD38RMFI values (B) percentage values for $\mathrm{CD}_{19}{ }^{+} \mathrm{CD} 34^{+}$(left) and CD $34^{+}{ }^{+}$D $38^{\text {low }}$ cells (right) of total $\mathrm{CD} 4^{+}$population in samples from patients with a range of conditions causing cytopenia including immune causes and/or splenomegaly (pathological controls, triangles) $(n=60)$. Patients with immune thrombocytopenia, hemolytic anemia and liver disease are shown as black bordered red triangles; MDS with a blast count of $<5 \%(n=63)$ (closed circles) including subgroup of MDS patients without ringed sideroblasts and/or an abnormal karyotype $(n=32)$ (red circles); MDS RAEB $(n=27)$ (triangles); AML $(n=31)$ (diamonds) and MPD/CMML $(n=11)$ (open circles). The clinical characteristics are set out in the Online Supplementary Table S2. CD34 ${ }^{+}$cells were defined by standard serial gating (CD34 ${ }^{+} S^{\text {low }}$ followed by CD34 $4^{+}$CD $\left.45^{\text {low }}\right)$. CD38RMFI=CD38PE-MFI divided by MFI of isotype control-PE staining. Horizontal bars are means and the SEM for each sample group is shown. The number of samples $(n=)$ in each group are shown in (A). Dotted line in (A) shows threshold value of CD38PE-RMFI defined by receiver-operator characteristic curve that in this cohort diagnoses low-grade MDS with $95 \%$ sensitivity $(95 \%$ confidence interval, $87-99 \%)$ and $92 \%$ specificity (95\% confidence interval, 82-97\%). 


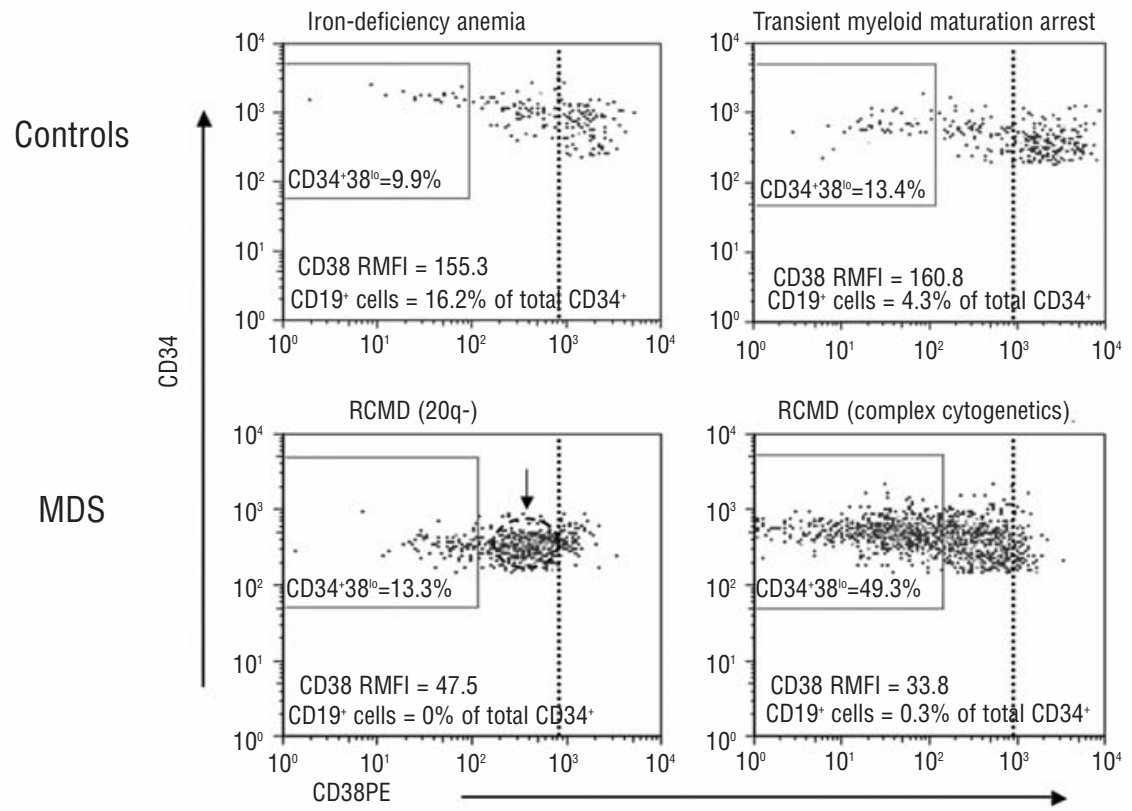

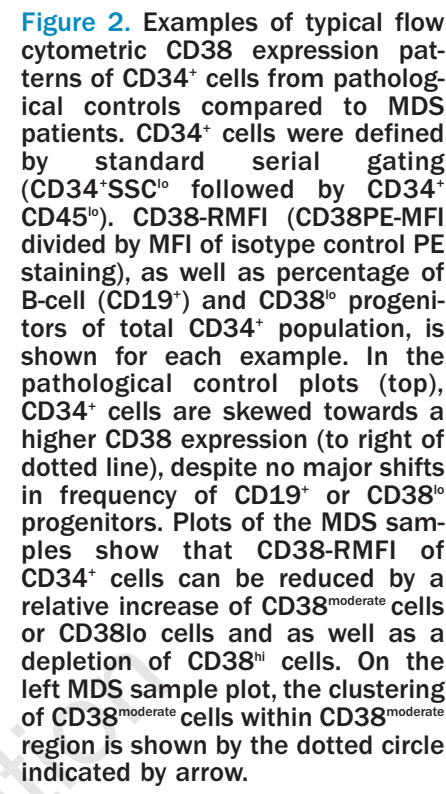

Figure 2. Examples of typical flow cospression patical controls compared to MDS patients. $\mathrm{CD} 4^{+}$cells were defined standard serial gating $\mathrm{CD}^{\circ 5^{\circ}}{ }^{\circ}$. CD38-RMFI (CD38PE-MFI divided by MFI of isotype control PE staining), as well as percentage of B-cell $\left(\right.$ CD19 $\left.^{+}\right)$and CD38 ${ }^{10}$ progenitors of total $\mathrm{CD}^{3} 4^{+}$population, is shown for each example. In the pathological control plots (top), cells are skewed towards a higher CD38 expression (to right of dotted line), despite no major shifts in ples show that CD38-RMFI of $\mathrm{CD}^{\circ} 4^{+}$cells can be reduced by a relative increase of $C D 38^{\text {moderate }}$ cells cells and as well as a depletion of $C D 38^{\text {hi }}$ cells. On the of CD38 ${ }^{\text {moderate }}$ cells within CD38 $8^{\text {moderate }}$ indicated by arrow. reproducible. Large numbers of nucleated bone marrow cells $\left(>0.5 \times 10^{6}\right)$ were acquired to provide an accurate analysis of CD38 RMFI in low frequency CD34 ${ }^{+}$subsets. CD38 $\mathrm{RMFI}$ in $\mathrm{CD}^{+}{ }^{+}$cells was lower in MDS patients (mean=27.6; range: 5.6-51.6) than normal controls (mean=58; range: 33.3-78.8). The difference in CD38 RMFI between $\mathrm{MDS}$ and pathological controls was even more striking, with no overlap in the range of values (MDS mean=27.6; range: 5.6-51.6 vs. pathological controls mean=194.5; range: 60.9-573). The low CD38 RMFI on $\mathrm{CD}^{+}{ }^{+}$cells does not simply result from an excess of immature $\mathrm{CD} 34^{+} \mathrm{CD} 38^{10 /-}$ progenitors, as when these were excluded from analysis by gating only the CD34+CD38 ${ }^{\text {moder }}$ ate/high cells (Online Supplementary Figure S1) CD38 RMFI values (of $\mathrm{CD} 34^{+} \mathrm{CD} 38^{\text {moderate/high }}$ subpopulations) remained reduced in $\mathrm{MDS}$ patients (mean=33.6; range: 16.1-62.9), compared to pathological (mean=210.1; range: 74.5-597.6) and normal controls (mean=65.1; range: 40.0-86.9) (data not shown).

We examined whether the relative frequencies of B-cell progenitors $\left(\mathrm{CD} 19^{+} \mathrm{CD} 34^{+}\right)$contributed to skewing of CD38 RMFI. Predictably, the percentage of CD19+ $\mathrm{CD} 34^{+} /$total $\mathrm{CD}^{+} 4^{+}$was reduced in MDS patients (mean=3.7\%; range: $0.3-14.8 \%$ ) compared to normal controls (mean $=13.4 \%$; range: $2.7-29.7 \%$ ) and pathological controls (mean $=12.9$; range: 0.1-37.7\%) (Online Supplementary Figure $S 1 B$ ). However, even in this small cohort, 4/10 pathological controls had reduced B-cell progenitors $(<1 \%)$, suggesting that this single parameter is not specific to diagnose MDS.

In this initial MDS patient cohort, skewed decrease of CD38 expression on CD34+ cells could not simply be attributed to decreased frequency of B-cell progenitors and/or $\mathrm{CD} 34^{+} \mathrm{CD} 38^{\text {hi }}$ precursors.

Since a reduction in the CD38 RMFI of CD34+ cells might distinguish $\mathrm{MDS}$ patients from those with non-clonal cytopenias, we tested the clinical use of this parameter in a larger prospective patient cohort: $90 \mathrm{MDS}$ patients (63
RA/RCMD and MDS-U; 27 RAEB-I and RAEB II) diagnosed by morphological review, clinical follow-up of at least six months and/or cytogenetic findings and 60 pathological control patients, the majority of whom had nonclonal cytopenias where the diagnosis was equivocal and MDS was considered as part of the differential diagnosis (e.g. anemia with dysplastic change but also mild renal impairment; pancytopenia with dysplasia in a patient with infection) (clinical details of these patients shown in Online Supplementary Table S2). Samples from AML patients $(n=31$, two thirds were $>55$ years) and CMML/MPD (excluding CML) ( $\mathrm{n}=11$ ) were also included (Online Supplementary Table S2). Samples in this cohort were routine clinical samples received and processed in a high throughput clinical laboratory using a four-color diagnostic antibody panel. Results from this cohort confirmed reduced CD38 RMFI of $\mathrm{CD}^{+} 4^{+}$cells in MDS samples with a clear separation between the majority of MDS and control samples (Figure 1A), despite an overlap in percentage values for $\mathrm{CD} 19^{+} \mathrm{CD} 34^{+}$and $\mathrm{CD} 34^{+} \mathrm{CD} 38^{\mathrm{lo} /}$ progenitors (Figure $1 \mathrm{~B}$ ). There was no discernable difference between the subgroup of MDS without an abnormal karyotype and/or ringed sideroblasts $(n=32)$ compared to the subgroup with an abnormal karyotype and/or ringed sideroblasts $(n=31)$ (Figure 1). This assay appears robust since it could be applied in a busy clinical laboratory that used different antibody clones/fluorochromes from those used by the independent laboratory analyzing cohort 1 . In MDS samples without excess blasts the RMFI of CD38 (mean=67.2; range 10.6-146.2) was reduced compared to pathological controls (mean=163.9; range 67.6-299). Importantly, a threshold value of CD38 PE RMFI (Figure 1A) defined by receiver-operator characteristic curve diagnosed low-grade MDS with 95\% sensitivity (95\% confidence interval, 87 $99 \%$ ), $92 \%$ specificity ( $95 \%$ confidence interval, $82-97 \%$ ), positive predictive value of $90.8 \%$ and negative predictive value of $91.5 \%$. Five of 6 control samples with lowest CD38 RMFI values were from patients with immune 
thrombocytopenia, hemolytic anemia, and severe liver disease. These diagnoses may, therefore, be more likely to produce false positives. Of the $3 \mathrm{MDS}$ samples over threshold value, the highest value was from a MDS patient responding well to erythropoietin which may have affected the nature of $\mathrm{CD}^{+} 4^{+}$cells in this patient. CD34 $4^{+}$cells from RAEB patients displayed an even greater reduction in CD38 RMFI but over half of these patients also had a high frequency of $\mathrm{CD} 34^{+} \mathrm{CD} 38^{\mathrm{lo} /-}$ progenitors (Figure 1). This is consistent with a previous study that specifically showed an increase in $\mathrm{CD}^{3} 4^{+} \mathrm{CD} 38^{-}$cells in high-risk and not lowrisk $\mathrm{MDS}^{8}$ Similar results were seen in AML samples (Figure 1).

The flow cytometric profile of CD38 expression on CD34+ blasts in both MDS patient cohorts revealed several patterns. These include: (i) clustering of some CD34 cells in the CD38moderate/low region (Figure 2), occasionally aberrant expression of CD34; (ii) shift of a proportion of $\mathrm{CD}_{3} 4^{+}$to $\mathrm{CD} 38^{\text {low/- }}$ region, most frequently seen in RAEB samples; and (iii) overall decrease in CD38 MFI without obvious clustering (data not shown). This contrasted with the pathological controls in which the CD34 ${ }^{+}$ population was often skewed towards higher CD38 expression (Figure 2).

This is the first study that has assessed the overall expression of $\mathrm{CD} 38$ on the total $\mathrm{CD}_{3} 4^{+}$population. Similar to previous studies, ${ }^{4,5}$ we have not found an increased frequency of $\mathrm{CD}_{34}{ }^{+} \mathrm{CD} 38^{-}$progenitors in lowgrade MDS, but despite this CD38 RMFI expression of the total $\mathrm{CD}_{3} 4^{+}$population from MDS patients is clearly decreased. However, as RMFI data might be modified for a variety of technical reasons, our findings do need to be confirmed by other laboratories.

An explanation for this finding may be that MDS results in an altered composition of myeloid progenitors in the $\mathrm{CD}_{34}{ }^{+} \mathrm{CD} 38^{+}$population. For example, common myeloid and granulocyte-macrophage progenitors have a lower CD38 expression than megakaryocyte-erythroid progenitors. ${ }^{16}$ Hence a relative imbalance in these progenitors could result in an overall decrease in the CD34 CD38 RMFI.

In summary, decreased CD38PE RMFI of the CD34+ population below a threshold value provides a potential simple single diagnostic flow cytometric measurement that separates MDS cases from those with non-clonal cytopenia and other pathological cases. This assay needs to be further validated in other centers but may be of widespread clinical utility in the diagnosis of MDS, especially those cases with a low blast count and a normal karyotype where an objective quantitative diagnostic test does not exist.

\section{Authorship and Disclosures}

NG, AS, PV and SF designed the experiments. AS, CC, $\mathrm{MD}, \mathrm{GS}$ and $\mathrm{RB}$ provided critical reagents and samples. NG, EM, W-KC, PR and SF performed the experiments. NG, PV and SF supervised the work. NG, EM, PV, and SF analyzed the results. SF made the figures. PV and SF wrote the paper and all the authors critically reviewed and revised it.

The authors reported no potential conflicts of interest.

\section{References}

1. Heaney ML, Golde DW. Myelodysplasia. N Engl J Med 1999:340: $1649-60$.

2. Nimer SD. Myelodysplastic syndromes. Blood 2008;111:4841-51.

3. Stetler-Stevenson M, Arthur DC, Jabbour N, Xie XY, Molldrem J, Barrett AJ, et al. Diagnostic utility of flow cytometric immunophenotyping in myelodysplastic syndrome. Blood 2001;98:979-87.

4. Del Canizo MC, Fernandez ME, Lopez A, Vidriales B, Villaron E, Arroyo JL, et al. Immunophenotypic analysis of myelodysplastic syndromes. Haematologica 2003;88: 4027.

5. Wells DA, Benesch M, Loken MR, Vallejo C, Myerson D, Leisenring WM, et al. Myeloid and monocytic dyspoiesis as determined by flow cytometric scoring in myelodysplastic syndrome correlates with the IPSS and with outcome after hematopoietic stem cell transplantation. Blood 2003;102:394-403.

6. Sternberg A, Killick S, Littlewood T, Hatton C, Peniket A, Seidl T, et al. Evidence for reduced B-cell progenitors in early (low-risk) myelodysplastic syndrome. Blood 2005;106: 2982-91

7. Della Porta MG, Malcovati L,
Invernizzi R, Travaglino E, Pascutto C, Maffioli $M$, et al. Flow cytometry evaluation of erythroid dysplasia in patients with myelodysplastic syndrome. Leukemia 2006;20:549-55.

8. Monreal MB, Pardo ML, Pavlovsky MA, Fernandez I, Corrado CS, Giere I, et al. Increased immature hematopoietic progenitor cells CD34+/CD38dim in myelodysplasia. Cytometry B Clin Cytom 2006; 70:63-70.

9. Ogata K, Kishikawa Y, Satoh C, Tamura H, Dan K, Hayashi A. Diagnostic application of flow cytometric characteristics of CD34+ cells in low-grade myelodysplastic syndromes. Blood 2006;108:1037-44.

10. Lorand-Metze I, Ribeiro E, Lima CS, Batista LS, Metze K. Detection of hematopoietic maturation abnormalities by flow cytometry in myelodysplastic syndromes and its utility for the differential diagnosis with nonclonal disorders. Leuk Res 2007;31: 147-55.

11. van de Loosdrecht AA, Westers TM, Westra AH, Drager AM, van der Velden VH, Ossenkoppele GJ. Identification of distinct prognostic subgroups in low- and intermediate1-risk myelodysplastic syndromes by flow cytometry. Blood 2008;111: 1067-77.

12. Matarraz S, Lopez A, Barrena S,
Fernandez C, Jensen E, Flores J, et al. The immunophenotype of different immature, myeloid and B-cell lineagecommitted CD34+ hematopoietic cells allows discrimination between normal/reactive and myelodysplastic syndrome precursors. Leukemia 2008:22:1175-83.

13. Satoh C, Dan K, Yamashita T, Jo R, Tamura H, Ogata K. Flow cytometric parameters with little interexaminer variability for diagnosing low-grade myelodysplastic syndromes. Leuk Res 2008;32:699-707.

14. Ribeiro E, Matarraz Sudon S, de Santiago M, Lima CS, Metze K, Giralt $M$, et al. Maturation-associated immunophenotypic abnormalities in bone marrow B-lymphocytes in myelodysplastic syndromes. Leuk Res 2006;30:9-16.

15. Maftoun-Banankhah S, Maleki A, Karandikar NJ, Arbini AA, Fuda FS, Wang HY, et al. Multiparameter flow cytometric analysis reveals low percentage of bone marrow hematogones in myelodysplastic syndromes. Am J Clin Pathol 2008;129: 300-8.

16. Edvardsson L, Dykes J, Olofsson T. Isolation and characterization of human myeloid progenitor populations--TpoR as discriminator between common myeloid and megakaryocyte/erythroid progenitors. Exp Hematol 2006;34:599-609. 